The Astrophysical Journal, 673:1218-1224, 2008 February 1

(C) 2008. The American Astronomical Society. All rights reserved. Printed in U.S.A.

\title{
FORMATION OF KUIPER BELT BINARIES
}

\author{
Hilke E. Schlichting and Re'em SARI \\ California Institute of Technology, MC 130-33, Pasadena, CA 91125; hes@astro.caltech.edu, sari@tapir.caltech.edu \\ Received 2007 July 19; accepted 2007 October 25
}

\begin{abstract}
The discovery that a substantial fraction of Kuiper Belt objects (KBOs) exists in binaries with wide separations and roughly equal masses has motivated a variety of new theories explaining their formation. Goldreich and colleagues proposed two formation scenarios: In the first, a transient binary is formed, which becomes bound with the aid of dynamical friction from the sea of small bodies $\left(L^{2} s\right.$ mechanism); in the second, a binary is formed by three-body gravitational deflection ( $L^{3}$ mechanism). Here, we accurately calculate the $L^{2} s$ and $L^{3}$ formation rates for sub-Hill velocities. While the $L^{2} s$ formation rate is close to previous order of magnitude estimates, the $L^{3}$ formation rate is about a factor of 4 smaller. For sub-Hill KBO velocities $\left(v \ll v_{\mathrm{H}}\right)$ the ratio of the $L^{3}$ to the $L^{2} s$ formation rate is $0.05\left(v / v_{\mathrm{H}}\right)$, independent of the small bodies' velocity dispersion, their surface density, or their mutual collisions. For super-Hill velocities $\left(v \gg v_{\mathrm{H}}\right)$ the $L^{3}$ mechanism dominates over the $L^{2} s$ mechanism. Binary formation via the $L^{3}$ mechanism competes with binary destruction by passing bodies. Given sufficient time, a statistical equilibrium abundance of binaries forms. We show that the frequency of long-lived transient binaries drops exponentially with the system's lifetime and that such transient binaries are not important for binary formation via the $L^{3}$ mechanism, contrary to Lee and colleagues. For the $L^{2} s$ mechanism we find that the typical time that transient binaries must last to form Kuiper Belt binaries (KBBs) for a given strength of dynamical friction, $D$, increases only logarithmically with $D$. Longevity of transient binaries (with lifetimes $15 \Omega^{-1}$ as suggested by Astakhov and colleagues) only becomes important for very weak dynamical friction (i.e., $D \lesssim 0.002$ ) and is most likely not crucial for KBB formation.
\end{abstract}

Subject headings: Kuiper Belt — planets and satellites: formation

\section{INTRODUCTION}

One of the many intriguing discoveries in the Kuiper Belt is that a substantial fraction of its largest members are binaries; 48 such systems are currently known (for a comprehensive review, see Noll et al. 2008). Broadly speaking, we can identify two classes of Kuiper Belt binaries (KBBs). The first class consists of small satellites around the largest Kuiper Belt objects (KBOs) and the second of roughly equal mass binaries with wide separations. The existence of the first class of binaries is most likely explained by the standard formation scenario involving a collision and tidal evolution, as has been proposed for the formation of the Moon and the Pluto-Charon system (Hartmann \& Davis 1975; Cameron \& Ward 1976; McKinnon 1989). This formation scenario fails however for the second class of KBBs, since it cannot account for their wide separations. This has motivated a variety of new theories for the formation of comparable-mass KBBs (e.g., Weidenschilling 2002; Goldreich et al. 2002; Funato et al. 2004; Astakhov et al. 2005; Lee et al. 2007). Weidenschilling (2002) proposed a new formation mechanism for KBBs consisting of a collision between two bodies inside the Hill sphere of a third. However, in the Kuiper Belt, gravitational scattering between the two intruders is about 100 times $^{1}$ more common than a collision. Binary formation by three-body gravitational deflection ( $L^{3}$ mechanism), as proposed by Goldreich et al. (2002), should therefore dominate over such a collisional formation scenario. Goldreich et al. (2002) proposed a second binary formation scenario: it consists of the formation of a transient binary, which becomes bound with the aid of dynamical friction from the sea

\footnotetext{
${ }^{1}$ For this estimate we used $\alpha \sim 10^{-4}$ and assumed that the velocity dispersion of the KBOs at the time of binary formation is less than their Hill velocity, see $\S 2$ for details.
}

of small bodies. This is called the $L^{2} s$ mechanism. Astakhov et al. (2005) and Lee et al. (2007) suggest that transient binaries that spend a long time in their mutual Hill sphere, near a periodic orbit, form the binaries in the $L^{2} s$ and $L^{3}$ mechanisms. We address and investigate the relative importance of these long-lived transient binaries for the $L^{2} s$ and $L^{3}$ formation mechanisms and find that they are most likely not significant for the overall binary formation in the Kuiper Belt. Finally, Funato et al. (2004) proposed a binary formation mechanism which involves a collision between two large KBOs which creates a small moon. An exchange reaction replaces the moon with a massive body with high eccentricity and large semimajor axis.

In this paper, we accurately calculate the $L^{2} s$ and $L^{3}$ formation rates for sub-Hill KBO velocities and discuss how these rates are modified for super-Hill velocities. This allows us to determine for which physical parameters and velocity regime each mechanism dominates the binary formation. Further, we calculate the frequency of long-lived transient binaries and assess their importance for the overall KBB formation.

Our paper is structured as follows. In $\S 2$ we outline our assumptions, explain our choice of parameters, and define variables that are used throughout this paper. We calculate the $L^{3}$ and $L^{2} s$ formation rates for sub-Hill KBO velocities in $\S 3$ and $\S 4$, respectively. We compare the $L^{2} s$ and $L^{3}$ formation rates in the sub-Hill velocity regime in $\S 5$. In $\S 6$ we discuss how these formation rates are modified for super-Hill KBO velocities. The frequency of long-lived transient binaries and their significance for the overall KBB formation is calculated in $\S 7$. Summary and conclusions follow in $\S 8$.

\section{DEFINITIONS AND ASSUMPTIONS}

The Hill radius denotes the distance from a KBO at which the tidal forces due to the Sun and the gravitational force due to the 
$\mathrm{KBO}$, both acting on a test particle, are in equilibrium. It is given by

$$
R_{\mathrm{H}} \equiv a\left(\frac{M}{3 M_{\odot}}\right)^{1 / 3}
$$

where $a$ is the semimajor axis, $M$ is the mass of the $\mathrm{KBO}$, and $M_{\odot}$ is the mass of the Sun. We use the "two-group approximation" (Goldreich et al. 2002, 2004) which consists of the identification of two groups of objects, small ones, which contain most of the total mass with surface mass density $\sigma$, and large ones, which contain only a small fraction of the total mass with surface mass density $\Sigma \ll \sigma$. We assume $\sigma \sim 0.3 \mathrm{~g} \mathrm{~cm}^{-2}$, which is the extrapolation of the minimum-mass solar nebula to a heliocentric distance of 40 AU. Estimates from current Kuiper Belt surveys (Trujillo \& Brown 2003; Trujillo et al. 2001) yield $\Sigma \sim 3 \times$ $10^{-4} \mathrm{~g} \mathrm{~cm}^{-2}$ for KBOs with radii of $R \sim 100 \mathrm{~km}$. We use this value of $\Sigma$, assuming that $\Sigma$ during the formation of KBBs was the same as it is now. Our choice for $\Sigma$ and $\sigma$ is also consistent with results from numerical coagulation simulations by Kenyon \& Luu (1999).

Large bodies grow by the accretion of small bodies. Large KBOs viscously stir the small bodies, increasing the small bodies' velocity dispersion $u$. As a result, $u$ grows on the same timescale as $R$ provided that mutual collisions among the small bodies are not yet important. In this case, $u$ is given by

$$
\frac{u}{v_{\mathrm{H}}} \sim\left(\frac{\Sigma}{\sigma \alpha}\right)^{1 / 2} \sim 3,
$$

where $\alpha=R / R_{\mathrm{H}} \sim 10^{-4}$ at $40 \mathrm{AU}$ (Goldreich et al. 2002) and $v_{\mathrm{H}}$ is the Hill velocity of the large bodies which is given by $v_{\mathrm{H}}=$ $\Omega R_{\mathrm{H}}$, where $\Omega$ is the orbital frequency around the Sun. The velocity $v$ of large KBOs increases due to mutual viscous stirring, but is damped by dynamical friction from the sea of small bodies such that $v<u$. Balancing the stirring and damping rates of $v$ and substituting for $u$ from equation (2), we find

$$
\frac{v}{v_{\mathrm{H}}} \sim \alpha^{-2}\left(\frac{\Sigma}{\sigma}\right)^{3} \sim 0.1 .
$$

For our choice of parameters, we have sub-Hill KBO velocities during the epoch of formation of bodies with $R \sim 100 \mathrm{~km}$. In addition, we argue that $v$ could not have exceeded $v_{\mathrm{H}}$ significantly during satellite formation in the Kuiper Belt. If $v_{\text {esc }}>v>v_{\mathrm{H}}$, where $v_{\text {esc }}$ is the escape velocity from the large bodies, then the timescale for mutual collisions is

$$
\begin{aligned}
\tau_{\text {coll }} \sim & 0.13\left(\frac{\Sigma}{3 \times 10^{-4} \mathrm{~g} \mathrm{~cm}^{-2}}\right)^{-1}\left(\frac{\rho}{1 \mathrm{~g} \mathrm{~cm}^{-3}}\right)\left(\frac{R}{100 \mathrm{~km}}\right) \\
& \times\left(\frac{\alpha}{1 \times 10^{-4}}\right)\left(\frac{v}{v_{\mathrm{H}}}\right)^{2}\left(\frac{\Omega}{7.9 \times 10^{-10} \mathrm{~s}^{-1}}\right)^{-1} \mathrm{Gyr} .
\end{aligned}
$$

Equation (4) shows that the collision timescale among the largest $\operatorname{KBOs}(R>100 \mathrm{~km})$ would have been excessively long if $v \gg v_{\mathrm{H}}$ during satellite formation. The ubiquity of small satellites around KBOs, which have radii as large as $\sim 1000 \mathrm{~km}$, (Brown et al. 2006; Brown \& Suer 2007) and the Pluto-Charon system (Weaver et al. 2006) suggests that sub-Hill KBO velocities prevailed during their formation, since their origin is best explained by a giant impact (e.g., Stern et al. 2006; Brown et al. 2007). We therefore focus our work on the shear-dominated velocity regime $\left(v \ll v_{\mathrm{H}}\right)$. However, we discuss how our results would be modified if $v \gg v_{\mathrm{H}}$.

\section{3. $L^{3}$ FORMATION RATE}

A transient binary forms when two large KBOs penetrate each other's Hill sphere. This transient binary must lose energy in order to become gravitationally bound. In the $L^{3}$ mechanism the excess energy is carried away by an encounter with a third massive body. We calculate the binary formation rate via the $L^{3}$ mechanism in the shear-dominated velocity regime. Since the growth of inclinations is suppressed in the shear-dominated velocity regime, the disk of KBOs is effectively two-dimensional (Wetherill $\&$ Stewart 1993; Rafikov 2003; Goldreich et al. 2004). We therefore restrict this calculation to two dimensions. As an initial condition, we assume that all bodies are on circular orbits. We chose to work in the rotating frame with the $x$-axis pointing radially outward and the $y$-axis in the prograde direction. For a gravitational deflection of three equal-mass bodies, the $L^{3}$ formation rate per body is

$$
\begin{aligned}
F R_{L^{3}}= & \int_{\gamma=-\infty}^{\infty} \int_{b_{2}>b_{1}}^{\infty} \int_{b_{1}=0}^{\infty}\left[\frac{\Sigma}{(4 \pi / 3) \rho R^{3}}\right]^{2} \\
& \times \frac{3}{2} b_{1} \Omega F_{L^{3}}\left(b_{1}, b_{2}, \gamma\right) d b_{1} d b_{2} d \gamma
\end{aligned}
$$

where $\Sigma /\left(4 \pi \rho R^{3} / 3\right)$ is the surface number density of the KBOs, $b_{1}$ and $b_{2}$ are the relative initial separations in the $x$-direction between bodies 1 and 2 and bodies 1 and 3 , respectively, and $\gamma$ is the offset in the $y$-direction body 3 would have when bodies 1 and 2 would encounter each other had their relative velocity been solely due to the Kepler shear of the disk: $3 b_{1} \Omega / 2$. Finally, $F_{L^{3}}\left(b_{1}, b_{2}, \gamma\right)$ is a function that takes on the value 1 if the encounter resulted in the formation of a binary between any two of the three KBOs involved and 0 otherwise. The choice of limits on the integrals in equation (5) ensures no double counting of the binaries. Equation (5) can be written as

$$
F R_{L^{3}}=A_{L^{3}}\left(\frac{\Sigma}{\rho R}\right)^{2} \alpha^{-4} \Omega,
$$

where

$$
\begin{aligned}
A_{L^{3}}=\left(\frac{27}{32 \pi^{2}}\right) & \int_{\gamma=-\infty}^{\infty} \int_{b_{2}>b_{1}}^{\infty} \int_{b_{1}=0}^{\infty} F_{L^{3}}\left(b_{1}, b_{2}, \gamma\right) \\
& \times\left(\frac{b_{1}}{R_{\mathrm{H}}}\right)\left(\frac{d b_{1}}{R_{\mathrm{H}}}\right)\left(\frac{d b_{2}}{R_{\mathrm{H}}}\right)\left(\frac{d \gamma}{R_{\mathrm{H}}}\right) .
\end{aligned}
$$

Equation (6) agrees with the order of magnitude estimate of Goldreich et al. (2002) if we set $A_{L^{3}}=1$. It is the value of the constant $A_{L^{3}}$ we determine here. Since we are interested in close encounters among the KBOs, their interaction is well described by Hill's equations (Hill 1878; Goldreich \& Tremaine 1980; Petit \& Henon 1986) that we modify to include three equal-mass bodies besides the Sun. The equations of motion, with length scaled by $R_{\mathrm{H}}$ and time by $\Omega^{-1}$, for body 1 are given by

$$
\begin{aligned}
\ddot{x}_{1}-2 \dot{y}_{1}-3 x_{1}= & -\frac{3\left(x_{1}-x_{2}\right)}{\left[\left(x_{1}-x_{2}\right)^{2}+\left(y_{1}-y_{2}\right)^{2}\right]^{3 / 2}} \\
& -\frac{3\left(x_{1}-x_{3}\right)}{\left[\left(x_{1}-x_{3}\right)^{2}+\left(y_{1}-y_{3}\right)^{2}\right]^{3 / 2}},
\end{aligned}
$$




$$
\begin{aligned}
\ddot{y}_{1}+2 \dot{x}_{1}= & -\frac{3\left(y_{1}-y_{2}\right)}{\left[\left(x_{1}-x_{2}\right)^{2}+\left(y_{1}-y_{2}\right)^{2}\right]^{3 / 2}} \\
& -\frac{3\left(y_{1}-y_{3}\right)}{\left[\left(x_{1}-x_{3}\right)^{2}+\left(y_{1}-y_{3}\right)^{2}\right]^{3 / 2}} .
\end{aligned}
$$

The subscripts 1, 2, and 3 label the $x$-and $y$-coordinates of KBO 1,2 , and 3 , respectively. Similar equations of motion can be obtained for bodies 2 and 3. The function $F_{L^{3}}\left(b_{1}, b_{2}, \gamma\right)$ is calculated by numerically integrating the equations of motion. A binary formation event is detected in the following way. The equations of motion of the three bodies are integrated until a time that corresponds to a separation of at least $30 R_{\mathrm{H}}$ between all three bodies (after their conjunction), assuming that their relative velocity is solely due to their Keplerian sheer (i.e., ignoring the actual gravitational interaction between the bodies), plus an additional time of $120 \Omega^{-1}$. If after this time the separation between two bodies is still less than $3 R_{\mathrm{H}}$, a binary is considered to have formed. We chose a separation of $3 R_{\mathrm{H}}$ instead of $R_{\mathrm{H}}$ to allow for binary orbits that reach slightly outside $R_{\mathrm{H}}$. Numerical integrations are terminated early if the separation between KBOs becomes less than $10^{-4} R_{\mathrm{H}}$ and these events are not counted toward the binaries formed. This serves two purposes. First of all, $10^{-4} R_{\mathrm{H}}$ roughly corresponds to the separation at which physical collisions occur in the Kuiper Belt. Second, by introducing a minimum separation, we prevent divergence in the equations of motion. This cutoff limits, strictly speaking, the validity of the value of $A_{L}^{3}$ calculated here to binary formation at heliocentric distances of $\sim 40 \mathrm{AU}$, since the separation in units of $R_{\mathrm{H}}$, corresponding to collisions among the KBOs, is inversely proportional to the heliocentric distance. In order to determine $A_{L^{3}}$ we need to cover the three-dimensional parameter space spanned by $b_{1}, b_{2}$, and $\gamma$. We chose a spacing of $0.1 R_{\mathrm{H}}$ for all three parameters; $12.5 R_{\mathrm{H}}$ is chosen as the upper limit for $b_{1}$ and $b_{2}$, and the upper limit for $|\gamma|$ is $25 R_{\mathrm{H}}$. The given limits and resolution require numerical integrations of $\sim 4 \times 10^{6}$ orbits. We obtain

$$
A_{L^{3}}=0.28 \pm 0.01
$$

where 0.01 is the estimated Poisson error. We repeated the calculation for $A_{L^{3}}$ with randomly chosen grid points for $b_{1}, b_{2}$, and $\gamma$ and the same number of numerical integrations and confirmed that the value of $A_{L^{3}}$ is insensitive to the grid points chosen. The value of $A_{L^{3}}$ tends to 0.35 in the limit that the bodies are treated as point masses (i.e., the limit that the cutoff tends to zero). We use $A_{L^{3}}=0.28$, since it corresponds to the physically relevant situation in the Kuiper Belt. This yields a binary formation rate of

$F R_{L^{3}}=(6.3 \pm 0.2) \times 10^{-8}\left(\frac{\Sigma}{3 \times 10^{-4} \mathrm{~g} \mathrm{~cm}^{-2}}\right)^{2}\left(\frac{\rho}{1 \mathrm{~g} \mathrm{~cm}^{-3}}\right)^{-2}$

$\times\left(\frac{R}{100 \mathrm{~km}}\right)^{-2}\left(\frac{\alpha}{1 \times 10^{-4}}\right)^{-4}\left(\frac{\Omega}{7.9 \times 10^{-10} \mathrm{~s}^{-1}}\right) \mathrm{yr}^{-1}$,

which is smaller by $1 / A_{L^{3}} \sim 4$ than the order of magnitude estimate of Goldreich et al. (2002).

\section{4. $L^{2} s$ FORMATION RATE}

So far, we have only considered binary formation due to an encounter with a third body that carries away the excess energy. However, binary formation might also occur due to dynamical friction generated by the sea of small bodies $\left(L^{2} s\right.$ mechanism). The random velocity of large KBOs is damped due to gravitational interactions with many small bodies. Since it is not feasible to examine the interactions with each small body individually, their net effect is modeled by an averaged force which acts to damp the large KBOs' noncircular velocity. We parameterize the strength of the damping by a dimensionless quantity $D$ defined as the fractional decrease in noncircular velocity due to dynamical friction over a time $\Omega^{-1}$,

$$
D \sim \frac{\sigma}{\rho R}\left(\frac{u}{v_{\mathrm{H}}}\right)^{-4} \alpha^{-2} \sim \frac{\Sigma}{\rho R} \alpha^{-2}\left(\frac{v}{v_{\mathrm{H}}}\right)^{-1} .
$$

The first expression is simply an estimate of dynamical friction by the sea of small bodies assuming $u>v_{\mathrm{H}}$. The second expression describes the mutual excitation among the large KBOs for $v \ll v_{\mathrm{H}}$. These two expressions can be equated, since the stirring among the large KBOs is balanced by the damping due to dynamical friction. In fact, if $v$ is defined as the product of the median eccentricity and the orbital velocity, we can calculate the exact relationship between $D$ and $\left(v / v_{\mathrm{H}}\right)$, since the velocity distribution in the sheardominated velocity regime has been fully determined (see Collins $\&$ Sari 2006; Collins et al. 2007). Defining $v$ as the product of the median eccentricity and the orbital velocity, we obtain

$$
D=4.1 \frac{\Sigma}{\rho R} \alpha^{-2}\left(\frac{v}{v_{\mathrm{H}}}\right)^{-1} \text {. }
$$

For $\rho \sim 1 \mathrm{~g} \mathrm{~cm}^{-3}$ and our estimates for $\left(v / v_{\mathrm{H}}\right), \Sigma$, and $R$ from $\S 2$, we find $D \sim 0.12$. We calculate the binary formation rate for equalmass bodies via the $L^{2} s$ mechanism in the shear-dominated velocity regime. As in $\S 3$, we restrict this calculation to two dimensions with circular motion as an initial condition for the large KBOs and use the same coordinate system as in $\S 3$. The binary formation rate per body via the $L^{2} s$ mechanism can be written as

$$
F R_{L^{2} s}=\int_{b=0}^{\infty}\left[\frac{\Sigma}{(4 \pi / 3) \rho R^{3}}\right] \frac{3}{2} b \Omega F_{L^{2} s}(D, b) d b,
$$

where $\Sigma /\left(4 \pi \rho R^{3} / 3\right)$ is the surface number density of the KBOs, $b$ is the relative initial separation in the $x$-direction between the two KBOs, and $F_{L^{2} s}(D, b)$ is a function that takes on the value 1 if the encounter resulted in the formation of a binary for a given $D$ and $b$ and 0 otherwise. Equation (14) can be written as

$$
F R_{L^{2} s}=A_{L^{2} s} D\left(\frac{\Sigma}{\rho R}\right) \alpha^{-2} \Omega
$$

where

$$
A_{L^{2} s}=D^{-1}\left(\frac{9}{8 \pi}\right) \int_{b=0}^{\infty} F_{L^{2} s}(D, b)\left(\frac{b}{R_{\mathrm{H}}}\right)\left(\frac{d b}{R_{\mathrm{H}}}\right) .
$$

Goldreich et al. (2002) showed, using numerical integrations, that $F R_{L^{2} S}$ is indeed proportional to $D$. Here we want to determine the actual value of $A_{L^{2} s}$. In Hill coordinates the equations of motion of the two KBOs can be decomposed into their center of mass motion and their relative motion with respect to one another. The relative motion of two equal-mass KBOs, including the dynamical friction term, is governed by

$$
\begin{gathered}
\ddot{x}-2 \dot{y}-3 x=-\frac{6 x}{\left(x^{2}+y^{2}\right)^{3 / 2}}-D \dot{x}, \\
\ddot{y}+2 \dot{x}=-\frac{6 y}{\left(x^{2}+y^{2}\right)^{3 / 2}}-D(\dot{y}+1.5 x) .
\end{gathered}
$$


where $x$ and $y$ correspond to the relative separation between the two KBOs in the $x$ - and $y$-direction, respectively. Again, length has been scaled by $R_{\mathrm{H}}$ and time by $\Omega^{-1}$. Equations (17) and (18) are integrated for different values of $D$ and impact parameters ranging from $2.2 R_{\mathrm{H}}$ to $3.2 R_{\mathrm{H}}$. Impact parameters outside this range result in a distance of closest approach between the two KBOs of more than $R_{\mathrm{H}}$. Figure 1 shows that the rate of binary formation is proportional to $D$. The value of $A_{L^{2} s}$, estimated from the line of best fit, is 1.4 . This yields a binary formation rate of

$$
\begin{aligned}
\mathrm{FR}_{L^{2} s}= & 1.3 \times 10^{-5}\left(\frac{D}{0.12}\right)\left(\frac{\Sigma}{3 \times 10^{-4} \mathrm{~g} \mathrm{~cm}^{-2}}\right) \\
& \times\left(\frac{\rho}{1 \mathrm{~g} \mathrm{~cm}^{-3}}\right)^{-1}\left(\frac{R}{100 \mathrm{~km}}\right)^{-1} \\
& \times\left(\frac{\alpha}{1 \times 10^{-4}}\right)^{-2}\left(\frac{\Omega}{7.9 \times 10^{-10} \mathrm{~s}^{-1}}\right) \mathrm{yr}^{-1}
\end{aligned}
$$

Using equation (12) we can retrieve the scalings of Goldreich et al. (2002). Although, we know the exact $L^{2} s$ formation rate for a given $D$ and have an exact expression for $D$ in terms of $v$ (see eq. [13]), the relation between $v$ and the actual physical parameters, i.e., the numerical coefficient in equation (3), which is needed for a precise value of $D$, is uncertain to a factor of order unity.

Contrary to claims by Astakhov et al. (2005) and Lee et al. (2007), the $L^{2} s$ mechanism does predict a mass-ratio selection. This can be seen from the first part of equation (12). For a given $u$, we have that $D \propto R^{3}$, since $v_{\mathrm{H}} \alpha^{-1 / 2} \sim v_{\text {esc }} \propto R$. Large KBOs experience stronger dynamical friction (larger $D$ ) than smaller ones. This is not at all surprising and is a general feature of dynamical friction (Chandrasekhar 1943; Binney \& Tremaine 1987). We can write $D=D_{0} M$, where $D_{0} \sim \sigma G^{2} / u^{4} ; D_{0}$ is a constant independent of the KBO mass for a given $\sigma$ and $u$. For two KBOs with masses $M_{1}$ and $M_{2}$, the position of body 1 essentially coincides with the center of mass of the two bodies provided that $M_{1} / M_{2} \gg 1$. In the limit that the KBOs' random velocity tends to zero and that $D_{0} M_{2} \ll 1$, we can place body 1 at the origin of the Hill coordinate system and treat the center of mass as stationary throughout the interaction. In this limit we find that the relative motion of the two KBOs is governed by

$$
\begin{gathered}
\ddot{x}-2 \dot{y}-3 x=-\frac{3 x}{\left(x^{2}+y^{2}\right)^{3 / 2}}-2 D_{0} M_{2} \dot{x}, \\
\ddot{y}+2 \dot{x}=-\frac{3 y}{\left(x^{2}+y^{2}\right)^{3 / 2}}-2 D_{0} M_{2}(\dot{y}+1.5 x),
\end{gathered}
$$

where length is scaled by $R_{\mathrm{H}}$ of KBO 1 and time is scaled by $\Omega^{-1}$. For extreme mass-ratio binaries the relevant strength of the dynamical friction that enters equations (20) and (21) is twice that acting on the small body (i.e., $2 D_{0} M_{2}$ ) and significantly less than that acting on the large body (i.e., $D_{0} M_{1}$ ). The $L^{2} s$ mechanism therefore favors the formation of comparablemass binaries from the largest available bodies over high-massratio ones. It is an open question whether this preference for comparable-mass binaries remains after the Kuiper Belt mass spectrum during their formation and their survival probability are accounted for.

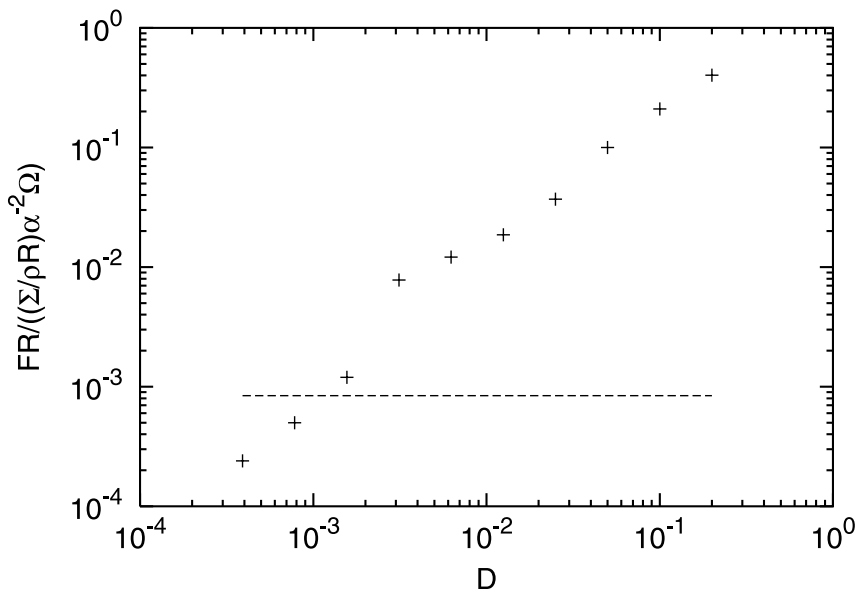

FIG. 1.-Binary formation rate as a function of dynamical friction strength $D$. The crosses correspond to the formation rate via the $L^{2} s$ mechanism, and the dashed horizontal line corresponds to the $L^{3}$ formation rate for $(\Sigma / \rho R) \alpha^{-2}=$ $3 \times 10^{-3}$. The $L^{2} s$ formation rate is proportional to $D$. In $\oint 4$ we estimate that $D \sim 0.12$, as a result of which $\mathrm{FR}_{L^{3}} / \mathrm{FR}_{L^{2} s} \sim 0.005$.

\section{COMPARISON OF $L^{2} s$ AND $L^{3}$ FORMATION RATES}

We are now able to compare the binary formation rates for the $L^{2} s$ and $L^{3}$ mechanisms for sub-Hill velocities. The ratio of the $L^{3}$ to $L^{2} s$ formation rates is

$$
\frac{\mathrm{FR}_{L^{3}}}{\mathrm{FR}_{L^{2} s}}=0.20 D^{-1} \frac{\Sigma}{\rho R} \alpha^{-2}=0.05 \frac{v}{v_{\mathrm{H}}},
$$

where we substituted for $D$ using the exact relationship from equation (13). It is remarkable that this expression depends explicitly only on $v / v_{\mathrm{H}}$ and is independent of what sets $D$. It is therefore independent of the velocity dispersion of the small bodies, their surface density, and the importance of collisions among the small bodies. We therefore conclude that for $v \ll v_{\mathrm{H}}$, binaries in the Kuiper Belt formed primarily due to dynamical friction rather than three-body encounters. Figure 1 shows the $L^{2} s$ and $L^{3}$ formation rates as a function of $D$. For our estimate of $\left(v / v_{\mathrm{H}}\right) \sim 0.1$, we have that $\mathrm{FR}_{L^{3}} / \mathrm{FR}_{L^{2} s} \sim 0.005$.

\section{SUPER-HILL VELOCITY: $v \gg v_{\mathrm{H}}$}

Obviously, there is some uncertainty in what the actual values of $\sigma$ and $\Sigma$ were during binary formation. For a few times larger value of $\Sigma$ with $\sigma$ unchanged, we enter the regime in which $v>$ $v_{\mathrm{H}}$ (this can be seen from eq. [2]). Although it is rather unlikely that $v \gg v_{\mathrm{H}}$ during binary formation (see $\S 2$ ), we discuss here briefly how this would affect the $L^{2} s$ and $L^{3}$ formation rates.

For super-Hill velocities the velocity dispersion of the large bodies is still set by the balance between their mutual stirring and the damping due to dynamical friction generated by the sea of small bodies. Therefore, dynamical friction shrinks the orbit of a KBB with a mutual orbital velocity $v_{B}$ at a rate

$$
D \Omega \sim \frac{\Sigma}{\rho R} \alpha^{-2} \Omega\left(\frac{v}{v_{\mathrm{H}}}\right)^{-4}
$$

where we assume that $v_{B}<u$. For $v_{B} \lesssim v$, binaries are broken up by passing $\mathrm{KBOs}$ at a rate

$$
\mathcal{R}_{\text {break }} \sim \frac{\Sigma}{\rho R} \alpha^{-2} \Omega\left(\frac{v}{v_{\mathrm{H}}}\right)^{-2}\left(\frac{v_{B}}{v_{\mathrm{H}}}\right)^{-2} .
$$


The ratio of these two rates yields

$$
\frac{D \Omega}{\mathcal{R}_{\text {break }}} \sim\left(\frac{v_{B}}{v}\right)^{2}
$$

Since the ratio in equation (25) is $<1$ for $v_{B}<v$, we conclude that KBBs with separations $R_{B}>R_{\mathrm{H}}\left(v_{\mathrm{H}} / v\right)^{2}$ (i.e., KBBs with $v_{B}<v$ ) tend to be broken up by passing KBOs. Binaries with separations of $R_{\text {crit }}=R_{\mathrm{H}}\left(v_{\mathrm{H}} / v\right)^{2}$ or less tend to survive. The cross section for the $L^{3}$ mechanism is therefore reduced with respect to the subHill case. The probability of having a KBO within $R_{\text {crit }}$ of a given $\mathrm{KBO}$ is $(\Sigma \Omega) /\left(\rho R^{3} v\right) R_{\text {crit }}^{3}$, where $(\Sigma \Omega) /\left(\rho R^{3} v\right)$ is the volume number density of KBOs. The flux of KBOs into area $R_{\text {crit }}^{2}$ is $(\Sigma \Omega) /\left(\rho R^{3} v\right) v R_{\text {crit. }}^{2}$. The super-Hill formation rate for tight binaries with separations $\sim R_{\text {crit }}$, via the $L^{3}$ mechanism, is therefore (see also Noll et al. 2008)

$$
\mathrm{FR}_{L^{3}} \sim\left(\frac{\Sigma \Omega}{\rho R^{3} v}\right)^{2} v R_{\text {crit }}^{5} \sim\left(\frac{\Sigma}{\rho R}\right)^{2} \alpha^{-4}\left(\frac{v_{\mathrm{H}}}{v}\right)^{11} \Omega .
$$

In addition to tight binaries with separations of $R_{\text {crit }}$ and less, there exists a second class of binaries with larger separations. Binaries with separations $R_{B}>R_{\text {crit }}$ are constantly created and destroyed via the $L^{3}$ mechanism. KBBs can form from two KBOs that approach each other with relative velocity $v_{B} \lesssim v$ while a third KBO removes energy, through gravitational scattering, enabling the KBOs to get bound. Since we are selecting bodies with relative velocities $\sim v_{B}$ or less, the number of KBOs that can form binaries with separation $R_{B}=R_{\mathrm{H}}\left(v_{\mathrm{H}} / v_{B}\right)^{2}$ is reduced by $\sim\left(v_{B} / v\right)^{3}$. The formation rate for binaries with separation $R_{B}=R_{\mathrm{H}}\left(v_{\mathrm{H}} / v_{B}\right)^{2}$ is

$$
\mathrm{FR}_{L^{3}}\left(R_{B}>R_{\text {crit }}\right) \sim\left(\frac{\Sigma}{\rho R}\right)^{2} \alpha^{-4}\left(\frac{v_{\mathrm{H}}}{v}\right)^{6}\left(\frac{v_{\mathrm{H}}}{v_{B}}\right)^{5} \Omega .
$$

These wider binaries $\left(R_{B}>R_{\text {crit }}\right)$ have a higher formation rate compared to the tight ones which have a separation $\sim R_{\text {crit }}$. The ratio of the formation rate (eq. [27]) to the destruction rate (eq. [24]) yields an equilibrium abundance of binaries per KBO at any given time that is given by

$$
\frac{N_{\mathrm{KBB}}}{N_{\mathrm{KBO}}} \sim \frac{\Sigma}{\rho R} \alpha^{-2}\left(\frac{v_{\mathrm{H}}}{v}\right)^{4}\left(\frac{v_{\mathrm{H}}}{v_{B}}\right)^{3} .
$$

The number of binaries scales as $\left(R_{B} / R_{\mathrm{H}}\right)^{3 / 2}$. Binaries with separation $R_{B}$ are therefore $\left(R_{B} / R_{\text {crit }}\right)^{3 / 2} \sim\left(v / v_{B}\right)^{3}$ times more common than those with separation $R_{\text {crit }}$ provided there is sufficient time for the equilibrium to be established. The same statistical equilibrium abundance can be derived using phase-space arguments. The phase-space number density of KBOs is $(\Sigma \Omega) /\left(\rho R^{3} v^{4}\right)$. The phase-space volume corresponding to a binary separation $R_{B}$ and velocity $v_{B}$ is $R_{B}^{3} v_{B}^{3}=R_{\mathrm{H}}^{3} v_{\mathrm{H}}^{3}\left(v_{\mathrm{H}} / v_{B}\right)^{3}$. Multiplying the KBO phasespace number density by the binary phase-space volume yields a statistical equilibrium abundance per KBO of

$$
\frac{N_{\mathrm{KBB}}}{N_{\mathrm{KBO}}} \sim \frac{\Sigma \Omega}{\rho R^{3} v^{4}} R_{\mathrm{H}}^{3} v_{\mathrm{H}}^{3}\left(\frac{v_{\mathrm{H}}}{v_{B}}\right)^{3} \sim \frac{\Sigma}{\rho R} \alpha^{-2}\left(\frac{v_{\mathrm{H}}}{v}\right)^{4}\left(\frac{v_{\mathrm{H}}}{v_{B}}\right)^{3},
$$

which is in agreement with the binary abundance derived in equation (28). Whether any of these binaries would survive the dynamical excitation of the Kuiper Belt remains an open question.

The $L^{2} s$ mechanism fails in creating binaries with separations $\sim R_{\text {crit }}$, since dynamical friction is not able to dissipate sufficient energy for tight binaries to form. Dynamical friction is only able to assist in the formation of binaries with wide separations $\left(\sim R_{\mathrm{H}}\right)$ that form from KBOs that happen to approach each other with low relative velocities $\left(\sim v_{\mathrm{H}}\right)$. This reduces the number density of KBOs that can participate in binary formation by a factor of $\sim\left(v_{\mathrm{H}} / v\right)^{3}$. In this case, the $L^{2} s$ formation rate is given by

$$
\begin{aligned}
\operatorname{FR}_{L^{2} s}\left(R_{B} \sim R_{\mathrm{H}}\right) & \sim D\left(\frac{\Sigma}{\rho R}\right) \alpha^{-2}\left(\frac{v_{\mathrm{H}}}{v}\right)^{4} \Omega \\
& \sim\left(\frac{\Sigma}{\rho R}\right)^{2} \alpha^{-4}\left(\frac{v_{\mathrm{H}}}{v}\right)^{8} \Omega
\end{aligned}
$$

where we have substituted for $D$ from equation (23) in the last step. These wide binaries face the same fate as the wide ones formed via the $L^{3}$ mechanism in that they will be broken up quickly due to scattering of other large bodies. However, the $L^{2} s$ mechanism does not even contribute significantly to the binary equilibrium abundance calculated in equation (28), since $\mathrm{FR}_{L^{2} s}\left(R_{B} \sim R_{\mathrm{H}}\right) / \mathrm{FR}_{L^{3}}\left(R_{B} \sim R_{\mathrm{H}}\right) \sim\left(v_{\mathrm{H}} / v\right)^{2} \ll 1$. Therefore, the $L^{2} s$ mechanism does not play an important role in KBB formation if super-Hill velocities prevail.

In summary, the $L^{3}$ mechanism forms tight binaries that tend to be saved from breakup at a rate that is reduced by a factor of $\left(v_{\mathrm{H}} / v\right)^{11}$ compared to the sub-Hill case. In addition, the $L^{3}$ mechanism forms wider binaries $\left(R_{B}>R_{\text {crit }}\right)$, at a higher rate that is "only" reduced by a factor of $\left(v_{\mathrm{H}} / v\right)^{6}\left(v_{\mathrm{H}} / v_{B}\right)^{5}$ relative to the subHill rate. These wide binaries are constantly created and destroyed, leading to an equilibrium abundance of binaries that scales as $\left(R_{B} / R_{\mathrm{H}}\right)^{3 / 2}$. The $L^{2} s$ mechanism is not important if KBOs have super-Hill velocities.

\section{FREQUENCY OF LONG-LIVED TRANSIENT BINARIES AND THEIR SIGNIFICANCE FOR BINARY FORMATION}

Astakhov et al. (2005) propose that transient binaries that spent a time of $15 \Omega^{-1}(\sim 600$ yr at $40 \mathrm{AU})$ or longer in their mutual Hill sphere, near a periodic orbit, are responsible for binary formation in the $L^{2} s$ and $L^{3}$ mechanisms. Here, we determine how the frequency of long-lived transient binaries depends on the transient binary lifetime. This allows us to quantify the importance of long-lived transient binaries for the overall binary formation. Finally, we address the significance of long-lived transient binaries for the $L^{2} s$ and $L^{3}$ formation mechanisms.

\subsection{Frequency of Long-Lived Transient Binaries}

First, we assess how common long-lived transient binaries are. We integrate equations (17) and (18) without the dynamical friction term and determine the time $t_{3 R_{\mathrm{H}}}$ over which the separation between the two KBOs is less than $3 R_{\mathrm{H}}$ for all KBOs that approach one another to $R_{\mathrm{H}}$ and less. We chose to calculate the time the two KBOs spent with a separation of less than $3 R_{\mathrm{H}}$ to allow for orbits that reach slightly outside of $R_{\mathrm{H}}$ but return back to within $R_{\mathrm{H}}$ during the encounter. We integrate $\sim 10^{5}$ orbits in total with impact parameters ranging from $2.2 R_{\mathrm{H}}$ to $3.2 R_{\mathrm{H}}$. Impact parameters outside this range result in a distance of closest approach between the two KBOs of more than $R_{\mathrm{H}}$. As initial conditions, we assume that the orbits of the bodies are circular. Figure 2 shows that the frequency of transient binaries decreases exponentially with the transient binary lifetime, $t_{3 R_{\mathrm{H}}}$. The line of best fit yields a differential transient binary frequency, valid for $t_{3 R_{\mathrm{H}}} \gtrsim 1 \Omega^{-1}$, of

$$
\frac{d\left(\mathrm{FR}_{\mathrm{tb}}\right)}{d\left(t_{3 R_{\mathrm{H}}} \Omega\right)}=1.0 \times 10^{-\left(0.25 t_{3 R_{\mathrm{H}}} \Omega\right)} \frac{\Sigma}{\rho R} \alpha^{-2} \Omega .
$$




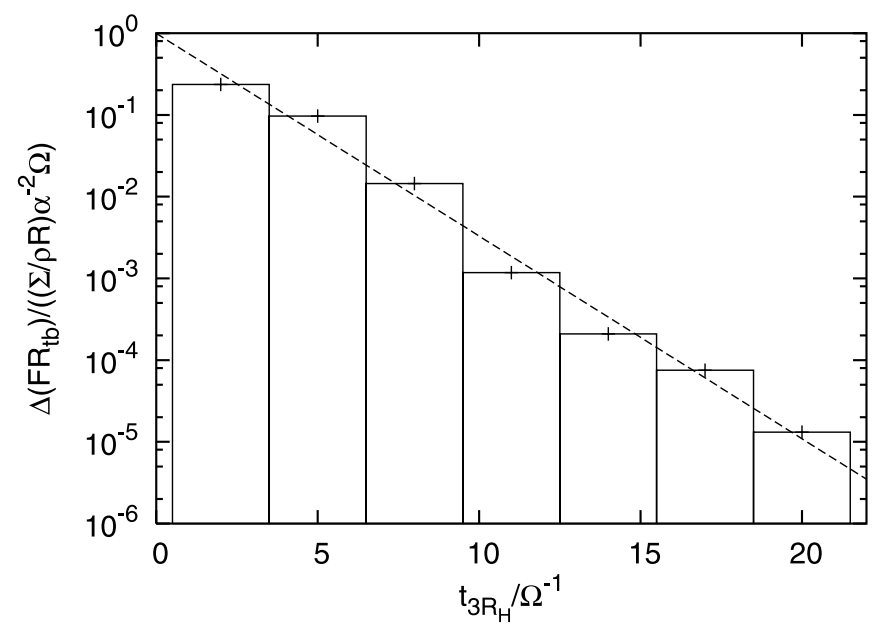

FIG. 2.-Differential transient binary frequency $\Delta\left(\mathrm{FR}_{\mathrm{tb}}\right)$ as a function of the transient binary lifetime $t_{3 R_{\mathrm{H}}}$ in the shear-dominated velocity regime, where $t_{3 R_{\mathrm{H}}}$ is the time the transient binary separation is less than $3 R_{\mathrm{H}}$. The frequency decreases exponentially with $t_{3 R_{\mathrm{H}}} \Omega$.

The frequency of transient binaries that spend a time of $\gtrsim 15 \Omega^{-1}$ with a separation of less than $3 R_{\mathrm{H}}$ is 3 orders of magnitude smaller than that of short-lived ones with $t_{3 R_{\mathrm{H}}} \gtrsim 3 \Omega^{-1}$. The analysis discussed here was carried out assuming that the KBOs approach each other with relative velocities $v_{\text {rel }} \ll v_{\mathrm{H}}$. Long-lived transient binaries do not exist for bodies that encounter each other at $v_{\text {rel }} \gg v_{\mathrm{H}}$. This can be understood by looking at the Jacobi constant. The Jacobi constant in Hill coordinates with length scaled by $R_{\mathrm{H}}$ and time by $\Omega^{-1}$ is given by

$$
C_{\mathrm{J}}=3 x^{2}-z^{2}+\frac{12}{\left(x^{2}+y^{2}+z^{2}\right)^{1 / 2}}-\dot{x}^{2}-\dot{y}^{2}-\dot{z}^{2} .
$$

KBOs that approach each other with $v_{\text {rel }} \gg v_{\mathrm{H}}$ at $R_{\mathrm{H}}$ will experience at most one encounter before they separate. Evaluation of their Jacobi constant at $R_{\mathrm{H}}$ yields that it is large and negative. In order to experience multiple encounters, KBOs must approach each other with $v_{\text {rel }} \sim v_{\mathrm{H}}$ at $R_{\mathrm{H}}$, which corresponds to $C_{\mathrm{J}}$ of order unity. Since the Jacobi constant is a conserved quantity, we can be sure that no long-lived transient binaries exist for KBOs that encounter each other at $v_{\text {rel }} \gg v_{\mathrm{H}}$. Long-lived transient binaries therefore offer no solution to the fine-tuning problem, contrary to claims by Lee et al. (2007). For KBOs with a given velocity distribution there always exist a few bodies that have $v_{\text {rel }}<v_{\mathrm{H}}$ even if $v \gg v_{\mathrm{H}}$. Such bodies can give rise to long-lived transient binaries in the same way that they can form wide binaries (see $\S 6$ for details), but the frequency of transient binaries due to such bodies is reduced by a factor of $\left(v_{\mathrm{H}} / v\right)^{4}$.

\subsection{Importance of Long-Lived Transient Binaries in the $L^{3}$ Formation Mechanism}

Lee et al. (2007) claim that the probability of binary formation from transient binaries with $t_{3 R_{\mathrm{H}}} \lesssim 2.5 \Omega^{-1}$ is extremely small, and they therefore include only transient binaries with $t_{3 R_{\mathrm{H}}} \geq 5 \Omega^{-1}$ in the main set of their integrations. However, their conclusion that the probability of binary formation from transient binaries with $t_{3 R_{\mathrm{H}}} \lesssim 2.5 \Omega^{-1}$ is extremely small is due to a bias in their initial conditions that discriminates against binary formation from transient binaries with $t_{3 R_{\mathrm{H}}} \lesssim 5 \Omega^{-1}$. The shortcoming of their analysis is due to the fact that they launch the third body from an initial separation $>38 R_{\mathrm{H}}$ when the first two KBOs come within a few $R_{\mathrm{H}}$ of each other. ${ }^{2}$ Since Lee et al. (2007) select the initial conditions for the third body such that it penetrates within $2.5 R_{\mathrm{H}}$, the largest impact parameter is $\sim 4.5 R_{\mathrm{H}}$. The minimum time it takes for the third body to come within a few $R_{\mathrm{H}}$ of the transient binary is therefore $\sim 38 R_{\mathrm{H}} /\left[(1.5) 4.5 R_{\mathrm{H}} \Omega\right] \sim 5.6 \Omega^{-1}$. The third body therefore only reaches the vicinity of the transient binary for $t_{3 R_{\mathrm{H}}} \geq 5.6 \Omega^{-1}$, but it is exactly this proximity of the third body that is required for binary formation by strong gravitational scattering. This explains why Lee et al. (2007) find such a small probability for binary formation by transient binaries with $t_{3 R_{\mathrm{H}}} \lesssim 5.6 \Omega^{-1}$. The range of impact parameters that lead to binary formation is comparable for short- and long-lived transient binaries. This means that the transient binary lifetime is the only advantage long-lived transient binaries have compared to short-lived ones, in terms of binary formation likelihood. However, as we show in $\S 7.1$, the frequency of transient binaries drops exponentially as a function of their lifetime. The ratio of the binary formation rate due to short-lived transient binaries $\left(t_{3 R_{\mathrm{H}}} \gtrsim 3 \Omega^{-1}\right)$ compared to that due to long-lived ones $\left(t_{3 R_{\mathrm{H}}} \gtrsim\right.$ $\left.15 \Omega^{-1}\right)$ is therefore

$$
\frac{\operatorname{FR}\left(t_{3 R_{\mathrm{H}}} \gtrsim 3 \Omega^{-1}\right)}{\operatorname{FR}\left(t_{3 R_{\mathrm{H}}} \gtrsim 15 \Omega^{-1}\right)} \sim \frac{3 \Omega^{-1}}{15 \Omega^{-1}} \frac{10^{-(0.25) 3}}{10^{-(0.25) 15}} \sim 200 .
$$

Although the binary formation rate scales linearly with transient binary lifetime, the frequency of transient binaries drops exponentially as a function of its lifetime. Therefore, long-lived transient binaries are not important for binary formation via the $L^{3}$ mechanism.

\subsection{Importance of Long-Lived Transient Binaries in the $L^{2} s$ Formation Mechanism}

In general, KBOs that spend a longer time in the Hill sphere lose more energy due to dynamical friction and are therefore more likely to be captured. However, they might not be responsible for the majority of the binary formation, if the frequency for longlived transient binaries is sufficiently small. To address this question we determine the typical time, $t_{\text {typ }}$, required for a transient binary to become bound with the aid of dynamical friction. We define $t_{\text {typ }}$ as the time it takes for $50 \%$ of all the KBOs that form a binary to become bound for a given strength of dynamical friction $D ; t_{\text {typ }}$ is measured from the point at which the relative separation between the two KBOs is less than $3 R_{\mathrm{H}}$. We determine $t_{\text {typ }}$ in the following way. First, we integrate the same equations as in $\S 4$ (i.e., eqs. [17] and [18]). We switch off the dynamical friction at different times and continue the evolution of the KBOs until $t=1000 \Omega^{-1}$. This process is repeated until we find the time for which dynamical friction has to act for $50 \%$ of all KBOs that form a binary to become bound. A transient binary is considered to have become bound when it remains a binary (i.e., relative separation $<3 R_{\mathrm{H}}$ ) until $t=1000 \Omega^{-1}$. We repeat this for different $D$ in order to reveal the relationship between $t_{\text {typ }}$ and $D$. Again, impact parameters are chosen to range from $2.2 R_{\mathrm{H}}$ to $3.2 R_{\mathrm{H}}$. Figure 3 shows that, for $D \gtrsim 0.002$, the typical time for permanent capture does not depend linearly on the strength of the dynamical friction $D$, but shows a weaker logarithmic dependence. The typical time $t_{\text {typ }}$ only ranges from $\sim 2 \Omega^{-1}$ for $D \sim 0.2$ to $\sim 10 \Omega^{-1}$ for $D \sim 0.002$. Furthermore, Figure 3 shows a noticeable break around $D \sim 0.001$; for $D \lesssim 0.001, t_{\text {typ }}$ increases significantly to $20 \Omega^{-1}$ and more. From this, we conclude that

\footnotetext{
2 The numerical values stated by Lee et al. (2007) are multiplied by a factor of $2^{1 / 3}$ to compensate for the different definitions of $R_{\mathrm{H}}$.
} 


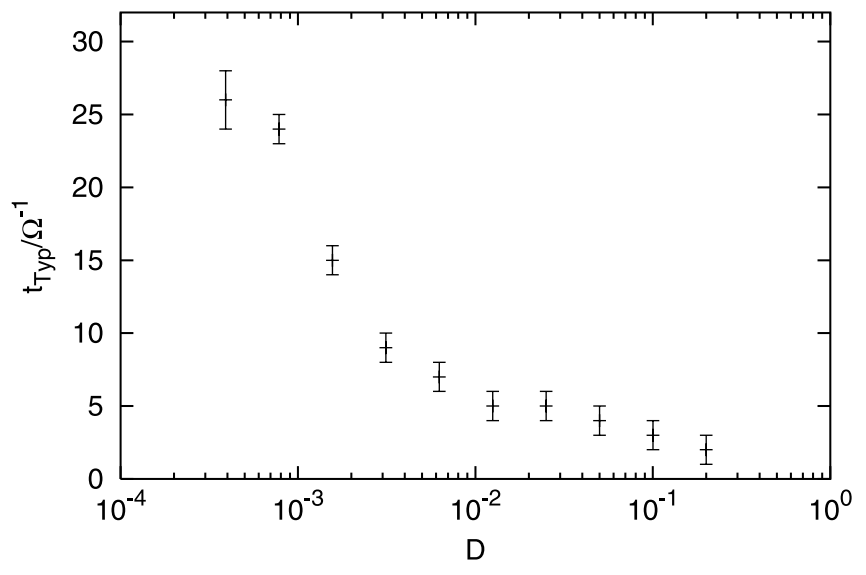

FIG. 3.- Variation of $t_{\mathrm{typ}}$, the time taken for $50 \%$ of binaries to get bound, plotted against strength of dynamical friction $D$. For about 2 orders of magnitude change in $D(D \sim 0.2$ to $D \sim 0.002)$, $t_{\text {typ }}$ only changes from $\sim 2 \Omega^{-1}$ to $\sim 10 \Omega^{-1}$. A rapid rise in $t_{\text {typ }}$ occurs for $D \lesssim 0.001$.

longevity of the transient binary (as discussed by Astakhov et al. [2005] with $t_{3 R_{\mathrm{H}}} \geq 15 \Omega^{-1}$ ) becomes only important for very weak dynamical friction (i.e., $D \lesssim 0.002$ ) and is most likely not crucial for $\mathrm{KBB}$ formation. In $\S 4$ we estimate $D \sim 0.12$, in which case longevity of transient binaries $\left(t_{3 R_{\mathrm{H}}} \geq 15 \Omega^{-1}\right)$ is unlikely to be a major requirement for binary formation.

\section{SUMMARY AND CONCLUSIONS}

We accurately determine the $L^{2} s$ and $L^{3}$ formation rates for sub-Hill velocities. We find that while the $L^{2} s$ formation rate is close to previous order of magnitude estimates, the $L^{3}$ formation rate is about a factor of 4 smaller. For $v \ll v_{\mathrm{H}}$, the ratio of the $L^{3}$ to the $L^{2} s$ formation rates is $\sim 0.05\left(v / v_{\mathrm{H}}\right)$ and is independent of what sets $D$. It is therefore independent of the velocity dispersion of the small bodies, their surface density, and the importance of collisions among the small bodies. For sub-Hill KBO velocities, binaries in the Kuiper Belt are formed primarily due to dynamical friction rather than three-body encounters. For super-Hill KBO velocities $\left(v \gg v_{\mathrm{H}}\right)$, the $L^{2} s$ mechanism becomes unimportant. The $L^{3}$ mechanism forms tight binaries that tend to be saved from breakup at a rate that is reduced by a factor of $\left(v_{\mathrm{H}} / v\right)^{11}$ compared to the sub-Hill case. In addition, the $L^{3}$ mechanism forms wider binaries $\left(R_{B}>R_{\text {crit }}\right)$, at a higher rate that is "only" reduced by a factor of $\left(v_{\mathrm{H}} / v\right)^{6}\left(v_{\mathrm{H}} / v_{B}\right)^{5}$ relative to the sub-Hill rate. These wide binaries are constantly created and destroyed, leading to an equilibrium abundance of binaries that scales as $\left(R_{B} / R_{\mathrm{H}}\right)^{3 / 2}$. Whether and how any of these wide binaries would survive the dynamical excitation of the Kuiper Belt remains an open question.

In addition, we determine the frequency of long-lived transient binaries. We show that the frequency of long-lived transient binaries drops exponentially with the system's lifetime for $v_{\text {rel }} \ll v_{\mathrm{H}}$. About 1000 transient binaries occur with $t_{3 R_{\mathrm{H}}} \gtrsim 3 \Omega^{-1}$ for each transient binary with $t_{3 R_{\mathrm{H}}} \gtrsim 15 \Omega^{-1}$. The long-lived transient binaries investigated by Astakhov et al. (2005) and Lee et al. (2007) are therefore very rare. Long-lived transient binaries are not important for binary formation via the $L^{3}$ mechanism, since the binary formation rate scales only linearly with transient binary lifetime, but the frequency of transient binaries drops exponentially as a function of its lifetime. Long-lived transient binaries do not exist for $v_{\text {rel }} \gg v_{\mathrm{H}}$. We show that the apparent shortage of binaries forming from short-lived transient binaries (i.e., $t_{3 R_{\mathrm{H}}} \lesssim 2.5 \Omega^{-1}$ ) found by Lee et al. (2007) can be explained by a bias in their initial conditions that discriminates against binary formation from transient binaries with $t_{3 R_{\mathrm{H}}} \lesssim 5 \Omega^{-1}$. Finally, to assess the importance of long-lived transient binaries in the $L^{2} s$ mechanism, we determine the typical time $t_{\text {typ }}$ required for a transient binary to become bound with the aid of dynamical friction. We show that longevity of the transient binary (as discussed by Astakhov et al. [2005] with $t_{3 R_{\mathrm{H}}} \geq 15 \Omega^{-1}$ ) only becomes important for very weak dynamical friction (i.e., $D \lesssim 0.002$ ). We estimate $D \sim 0.12$, in which case longevity of transient bina$\operatorname{ries}\left(t_{3 R_{\mathrm{H}}} \geq 15 \Omega^{-1}\right)$ is unlikely to be a major requirement for binary formation.

We thank Peter Goldreich for stimulating discussions and the anonymous referee for valuable comments that helped to clarify the manuscript. Some of the numerical calculations presented here were performed on Caltech's Division of Geological and Planetary Sciences Dell cluster. R. S. is an Alfred P. Sloan Fellow and a Packard Fellow.
Astakhov, S. A., Lee, E. A., \& Farrelly, D. 2005, MNRAS, 360, 401

Binney, J., \& Tremaine, S. 1987, Galactic Dynamics (Princeton: Princeton Univ. Press)

Brown, M. E., Barkume, K. M., Ragozzine, D., \& Schaller, E. L. 2007, Nature, 446, 294

Brown, M. E., \& Suer, T.-A. 2007, IAU Circ., 8812, 1

Brown, M. E., et al. 2006, ApJ, 639, L43

Cameron, A. G. W., \& Ward, W. R. 1976, Lunar Planet. Sci. Conf., 7, 120

Chandrasekhar, S. 1943, ApJ, 97, 255

Collins, B. F., \& Sari, R. 2006, AJ, 132, 1316

Collins, B. F., Schlichting, H. E., \& Sari, R. 2007, AJ, 133, 2389

Funato, Y., Makino, J., Hut, P., Kokubo, E., \& Kinoshita, D. 2004, Nature, 427, 518

Goldreich, P., Lithwick, Y., \& Sari, R. 2002, Nature, 420, 643 2004, ARA\&A, 42, 549

Goldreich, P., \& Tremaine, S. 1980, ApJ, 241, 425

\section{REFERENCES}

Hartmann, W. K., \& Davis, D. R. 1975, Icarus, 24, 504

Hill, G. W. 1878, Am. J. Math., 1, 5

Kenyon, S. J., \& Luu, J. X. 1999, AJ, 118, 1101

Lee, E. A., Astakhov, S. A., \& Farrelly, D. 2007, MNRAS, 379, 229

McKinnon, W. B. 1989, ApJ, 344, L41

Noll, K. S., Grundy, W. M., Chiang, E. I., Margot, J.-L., \& Kern, S. D. 2008, Icarus, in press (arXiv: 0711.1545)

Petit, J.-M., \& Henon, M. 1986, Icarus, 66, 536

Rafikov, R. R. 2003, AJ, 125, 942

Stern, S. A., et al. 2006, Nature, 439, 946

Trujillo, C. A., \& Brown, M. E. 2003, Earth Moon Planets, 92, 99

Trujillo, C. A., Jewitt, D. C., \& Luu, J. X. 2001, AJ, 122, 457

Weaver, H. A., et al. 2006, Nature, 439, 943

Weidenschilling, S. J. 2002, Icarus, 160, 212

Wetherill, G. W., \& Stewart, G. R. 1993, Icarus, 106, 190 